\title{
e-Migrinter
}

$11 \mid 2013$

Et l'immobilité dans la circulation?

\section{Migration et changement climatique en zone de montagne \\ $2^{\text {ème }}$ atelier de recherche, Institut de Géographie de Neuchâtel (Suisse) \\ Eleonora Guadagno et Raoul Kaenzig}

\section{OpenEdition}

\section{Journals}

\section{Édition électronique}

URL : https://journals.openedition.org/e-migrinter/276

DOI : $10.4000 /$ e-migrinter.276

ISSN : 1961-9685

\section{Éditeur}

UMR 7301 - Migrinter

\section{Édition imprimée}

Date de publication : 1 septembre 2013

Pagination : 198-200

ISSN : 1961-9685

Référence électronique

Eleonora Guadagno et Raoul Kaenzig, " Migration et changement climatique en zone de montagne », e-Migrinter [En ligne], 11 | 2013, mis en ligne le, consulté le 20 mai 2021. URL : http://

journals.openedition.org/e-migrinter/276 ; DOI : https://doi.org/10.4000/e-migrinter.276 
«Migration et changement climatique en zone de montagne ", $2^{\text {ème }}$ atelier de recherche, Institut de Géographie de Neuchâtel (Suisse), les 28 \& 29 mai 2013

\author{
Compte rendu par Eleonora Guadagno \& Raoul Kaenzig
}

A

vec les changements

climatiques, les régions montagneuses sont

considérées comme une des zones

les plus vulnérables, tout comme les déserts ou les espaces côtiers :

en effet, il est démontré que les perturbations environnementales qui provoquent des déplacements de populations en provenance des écosystèmes montagneux sont de plus en plus fréquentes. Cela est en premier lieu dû au fait que la mobilité a toujours représenté une stratégie de diversification du risque (qu'il soit environnemental ou bien économique) et de la vulnérabilité pour les communautés qui habitent les montagnes.
L'Atelier

Le deuxième atelier de recherche sur le thème "Migration et changement climatique dans les paysages montagneux", qui s'est tenu du 28 au 29 mai 2013 à l'Institut de Géographie de Neuchâtel, en Suisse, a vu la participation de chercheurs et doctorants travaillant sur le sujet et intéressés à partager leurs expériences empiriques et leurs approches théoriques.

L'atelier a été introduit par Etienne Piguet $^{1}$, qui a structuré les différentes sessions autour de plusieurs problématiques concernant la vie sociale, économique et culturelle des communautés touchés par les changements climatiques en région montagneuse : les communications se sont appuyées sur les expériences de terrains de recherche des chercheurs et des doctorants.

Dans le cadre d'une approche pluridisciplinaire, les sessions des deux journées ont mis en exergue les études empiriques de différentes zones géographiques.

Le retour d'expérience :

Les phénomènes migratoires augmenteront-tils sous l'influence du changement climatique? Quel est le rôle des facteurs environnementaux dans les migrations passées, présentes et futures dans les zones montagneuses?

${ }^{1}$ Professeur de Géographie à Institut de Géographie, Université de Neuchâtel. 
Cette première question nous incite à analyser les principaux facteurs liés aux migrations dites «environnementales ». Lors des différentes sessions, un débat est apparu autour de cette question, né des évidences empiriques dégagées des travaux de terrain.

En effet, les participants se sont entendus pour reconnaître la notion de multicausalité comme un élément incontournable dans l'étude de tout contexte migratoire. Des catastrophes lentes (désertification, fonte des glaciers, salinisation, etc.) pourraient affecter à long terme la durabilité de certaines activités humaines dans les zones montagneuses. Des exemples ont d'abord été fournis à partir des études du projet Rainfall $^{2}$ auprès de certains villages montagneux de la région du Kilimandjaro, en Tanzanie. Selon Tamer Afifi, les résultats de cette première étude montrent que la variation de l'intensité et de la fréquence des pluies qui sont liées aux changements climatiques, provoque des effets très négatifs sur la production agroalimentaire locale, générant une forte mobilité dans les communautés étudiées.

La migration actuelle est-elle, en quelque sorte "neutre 》 dans ses effets ou bien accroît-elle la vulnérabilité pour "ceux qui restent derrière $»^{3}$, c'est à dire ceux qui ne peuvent pas migrer? De plus, peut-on considérer qu'une communauté montagneuse soit plus résiliente face aux changements climatiques si elle est plus mobile et si la migration peut être envisagée comme stratégie d'adaptation?

Grâce aux exemples des Andes colombiennes et des Apennins italiens (développés respectivement par Vicente Anzellini et Eleonora Guadagno), il a été

\footnotetext{
2Soutenu par l'ONG CARE et l'Université des Nations Unies, cette étude analyse les interactions entre variabilité des précipitations, sécurité alimentaire et déplacements de populations dans huit pays en développement.

'L'expression anglaise "trapped population" est utilisée par R. Black dans le rapport Foresight Migration and Global Environmental Change Final Project Report (Government Office for Science, 2011).
}

possible d'analyser les effets des catastrophes soudaines (tremblement de terre, tsunami, inondation, etc.) sur différentes communautés et la vulnérabilité de ces dernières. Une attention particulière à été portée aux villages détruits par des glissements de terrain: si la réinstallation devient souvent inévitable, elle peut aussi provoquer des mécontentements, ainsi qu'un appauvrissement économique et social chez les communautés concernées.

La session a aussi introduit le lien entre migration et résilience. L'étude conduite dans la région de Panchase, au Népal (par Stéphanie Jaquet), essaie de comprendre comment la gestion des terres est affectée par les effets des changements climatiques et des désastres, ainsi que les mesures qui pourraient être mises en place pour augmenter la capacité de résilience des villageois. Au regard de la situation de la vallée de Gangotri, en Inde (présenté par Shrabana Datta), on peut analyser la manière dont la migration peut être un moyen pour faire face à la pénurie des récoltes. Évidemment, ceux qui ne peuvent pas migrer sont plus sujets à la vulnérabilité environnementale parce qu'ils sont obligés de rester dans les zones les plus fortement dégradées.

La seule étude - présentée lors de cette rencontre - à être entièrement basée sur une méthodologie quantitative a été conduite dans les Andes équatoriennes (par Raùl Vanegas). Elle vise à comprendre les dynamiques qui existent entre les pratiques agricoles, l'utilisation des terres et la perception de la dégradation.

Les communautés montagneuses sont souvent très dépendantes des centres urbains, qui sont, en outre, la principale destination de ces migrants. Les zones urbaines, à leur tour, sont très exposées aux risques causés par les changements climatiques. Quel rôle jouent les villes dans le contexte d'une migration provenant des zones de montagne et due aux changements climatiques? 
Le deuxième cas d'étude du projet Rainfall, conduit dans la zone de Huancayo au Pérou (et discuté par Andrea Milan), souligne l'importance de la distance entre les villes dans les dynamiques migratoires dans un contexte de désertification et de sécheresse. Les liens entre communautés montagneuses et centres urbains sont également importants dans le cas des Andes boliviennes (cas traité par Raoul Kaenzig) où la fonte des glaciers provoque une forte migration vers les villes de $\mathrm{La} \mathrm{Paz}$ et El Alto. La signification symbolique des glaciers est aussi très forte, dans la recherche concernant les implications anthropologique des changements climatiques, l'on analyse la perception de la population vis-à-vis des changements climatiques: dans la communauté Q'ero, dans les Andes Péruviennes (exemple développé par Geremia Cometti), il est possible, en effet, de reconnaître des modèles de mobilité qui lient facteurs matériels et symboliques.

\section{Pour conclure...}

Une synthèse mentionnant les principaux points de l'atelier a été effectuée par Richard Black ${ }^{4}$. Il a également présenté le cas du Portugal où il a étudié les liens entre la dégradation environnementale et le processus migratoire. Il a souligné l'importance de considérer l'environnement, comme un élément contextuel, mais aussi de le percevoir dans toutes ses variables économiques, sociales, démographiques et politiques. Pour cela, il apparaît essentiel d'analyser les facteurs environnementaux tout en considérant l'ensemble des dynamiques locales dans un cadre de changements globaux.
Eleonora Guadagno Doctorante en Géographie

Migrinter - UMR 7301

CNRS/Université de Poitiers guadagno.eleonora@gmail.com

Raoul Kaenzig Doctorant en Géographie Institut de Géographie Université de Neuchâtel raoul.kaenzig@unine.ch

4Professeur de Géographie humaine au Centre for Migration Research, Geography and International Development, University of Sussex. 